\title{
ON SECTION A OF PRINCIPIA MATHEMATICA $\dagger$
}

\author{
BY B. A. BERNSTEIN
}

1. Introduction. Section A of Whitehead and Russell's Principia Mathematica, which is concerned with the "theory of deduction" for "elementary" propositions, consists of "formal" (or "official") propositions and of "informal" (or "unofficial") statements. In previous papers, $\ddagger$ I discussed the theory of deduction when that theory is considered as given solely by the "formal" propositions. Huntington $§$ has recently given sets of postulates for "the algebra of logic" (or "Boolean algebra," or "the classic logic of classes," or "the logic of classes"); $\|$ and he obtained, in connection with these postulate-sets, two "informal" systems, consisting of "formal" and of "informal" propositions of Section A. I propose to bring out certain facts concerning Section A in view of Huntington's findings. My main results are that the "formal" Section A, as given by Huntington or by me, is derivable from the logic of classes; that this "formal" Section A is not the whole of the logic of classes; and that Huntington's "informal" systems are not adequate either for the classic logic of classes or for "the classic logic of propositions." "I

2. The "Formal" Section A Derivable from the Logic of Classes. Huntington's sixth set of postulates for Boolean algebra is expressed in terms of the following undefined ideas: a class $K$, a subclass $T$ of $K$, a binary operation + , and a unary operation'. In this set, Postulates 6.1, 6.2, 6.3, 6.4, 6.5, 6.6, 6.7 correspond

$\dagger$ Presented to the Society, March 18, 1933.

‡ See especially this Bulletin, vol. 37 (1931), pp. 480-488, and vol. 38 (1932), pp. 589-593. I shall refer to these papers later as Paper 1 and Paper 2 , respectively.

$\S$ See the Transactions of this Society, vol. 35 (1933), pp. 274-304. Later references to Huntington will imply this paper.

\|I I use these terms interchangeably for the general logic of classes as developed by Boole, modified by Schröder, and formulated postulationally by Huntington in his Sets of postulates for "the algebra of logic."

TI I use the term "the classic logic of propositions," or simply "the logic of propositions," for Schröder's A ussagenkalkiul as formulated in my Sets of postulates for the logic of propositions, Transactions of this Society, vol. 28 (1926), pp. $472-478$. 
respectively to my Principia propositions $\nmid 1.71,1.7,1.1,1.2$, $1.3,1.4,1.6$, except that my " $a=1$ " is replaced by " $a$ is in $T$." The sixth set of postulates thus contains all the (independent) primitive propositions underlying the "formal" Section A. Hence, the "formal" Section A, in Huntington's T-form, is derivable from the logic of classes.

Also, it can be verified $\ddagger$ that my Principia postulates 1.71 , 1.7, 1.1, 1.2, 1.3, 1.4, 1.6 can all be derived from Huntington's first set of postulates for Boolean algebra. Hence, the "formal" Section A, in my 1-form, is derivable from the logic of classes.

We thus find that, as far as the "formal" Section A is concerned, there is no proposition in it which is peculiar to the logic of propositions.

3. The "Formal" Section A not the Whole of the Logic of Classes. Besides Postulates 6.1-6.7 mentioned above, Huntington's sixth set of postulates also contains the following postulate.

Postulate 6.8. "If $T$ is a subclass having the five properties (6.3-6.7) just mentioned, then we have: If $a^{\prime}+b$ is in $T$, and $b^{\prime}+a$ is in $T$, then $a=b . "$

But Postulate 6.8 is independent of $6.1-6.7$, and Postulates 6.1-6.7, we saw, constitute the "formal" Section A. Hence, the "formal" Section A, in the T-form, is not the whole of the logic of classes.

I have shown, in another connection, $\S$ that one cannot derive from my 1 -form of the "formal" Section A the proposition $P$ following: $a^{\prime} \neq a$. But proposition $P$ is a proposition in the logic of classes. Hence, the "formal" Section A, in the 1-form, is not the whole of the logic of classes. We find then that the "formal" Section A alone, whether in the T-form of in the 1-form, is not adequate for the classic logic of classes.\|

$\dagger$ See Paper 1, loc. cit.

$\ddagger$ By using the following Boolean propositions: (a) 0,1 are unique; (b) $1+a=a+1=1$; (c) $a+a=a$; (d) $a^{\prime}+a=1$; (e) $(a+b)^{\prime}=a^{\prime} b^{\prime}$; (f) $(a+b)+c$ $=a+(b+c)$; (g) $a^{\prime} b+a=a+b$; (h) $a^{\prime} \neq a$; (i) $1^{\prime}=0,0^{\prime}=1$.

$\S$ In Paper 2, loc. cit.

|| It is then to be expected that in the derivation of the "the algebra of logic" in the Principia (Chapter *22), the authors would use primitives not found in Section A. This, indeed, is the case. Thus, in the proof of Postulate Ia of Huntington's first set of postulates for Boolean algebra, they use, in addition to the primitive propositions of Section A, also the following primitive propositions outside Section A: *9.1, *9.12, *9.13, *9.14, *9.15, *12.1. 
4. Inadequacy for Classes and for Propositions of Huntington's "Informal" System ( $K, \vee, \sim$, 三). Huntington (in Appendix I), constructs an "informal" system for Section A out of the Principia propositions $* 1.71, * 1.7, * 4.31, * 4.33, * 4.25, * 4.5$, $* 4.42, * 4.2, * 4.21, * 4.22$, together with certain "informal" statements in Section A regarding the sign $\equiv$. He identifies these propositions with the postulates of his fourth set, and then comes to the following conclusion: "If we accept the above mentioned informal statements as a valid part of the Principia, we have the following theorem.

TheOREM I. With respect to $(K, v, \sim, \equiv)$, the informal system of the Principia is a Boolean algebra."

Huntington, further, identifies the Principia proposition * 5.15 with a certain "informal" statement, and concludes: "If we accept the informal as well as the formal statements, we have

THEOREM II. With respect to $(K, \vee, \sim$, $\equiv)$, the informal system of the Principia is a Boolean algebra containing only two nonequivalent elements."

Now, if we examine the propositions that determine the system $(K, v, \sim, \equiv)$, we find that they are all satisfied when the class $K$ is empty. Hence the system $(K, \vee, \sim, \equiv)$ cannot yield such a proposition as the following.

$Q$. There is an element $u$ in $K$ such that $a u=a$ for every element $a$ in $K$.

But proposition $Q$ is a proposition in the classic logic of classes and in the classic logic of propositions. Hence, the system $(K, v, \sim, \equiv)$ is inadequate for the classic logic of classes and for the classic logic of propositions. $\dagger$

5. Inadequacy for Classes and for Propositions of Huntington's "Informal" System $\left(K, T,+,{ }^{\prime}\right)$. Huntington gives (in Appendix

$\dagger$ In view of the fact that $K$ may be empty, "only two," in Theorem II must be taken to mean "at mosi two." Theorem I seems to be an oversight. In identifying the postulates of his fourth set with corresponding Principia propositions, Huntington apparently overlooks the "trivial preliminary postulate" 4.0, demanding that $K$ have at least two elements. Also, in rewriting the Principia propositions $* 4.31, * 4.33, \cdots, * 4.42$, Huntington omits the sign " $\vdash$." (In rewriting *4.2 he retains " $\vdash . ")$ The Principia propositions used by Huntington form, it seems to me, a system $(K, \vee, \sim, \equiv, \vdash)$, not $(K, \vee, \sim, \equiv)$. 
II) a set of seven independent postulates, expressed in terms of $K, T,+,^{\prime}$ ( $K$ being the class of "elementary" propositions, and $T$ a subclass of "true" propositions in $K$ ), as basis for an "informal Principia system." The postulates are as follows:

Postulate 1. If $a$ and $b$ are in $K$, then $a+b$ is in $K$. [ $* 1.71$.]

Postulate 2. If $a$ is in $K$, then $a^{\prime}$ is in $K$. [*1.7.]

Postulate 3. If $a, b$, etc. are in $K$, then $b^{\prime}+(a+b)$ is in $T$. $[* 1.3$.

Postulate 4. If $a, b$, etc. are in $K$, then $(a+b)^{\prime}+(b+a)$ is in $T .[* 1.4$.

Postulate 5. If $a, b, c$, etc. are in $K$, then $\left(b^{\prime}+c\right)^{\prime}+\left\{(a+b)^{\prime}\right.$ $+(a+c)\}$ is in $T .[* 1.6$.

Postulate 6. If $a+b$ is in $T$, then at least one of the elements $a$ and $b$ is in $T$.

Postulate 7. If $a^{\prime}$ is in $T$, then $a$ is not in $T$.

Huntington observes that the first five of the above postulates correspond "precisely to the 'formal,' and the last two to 'informal' statements in the Principia." After deriving from the above postulates the Principia propositions $* 1.1, * 1.2, * 1.5$ (the remaining primitive propositions of Section A), he says: "Any system $\left(K, T,+,{ }^{\prime}\right)$ which satisfies Postulate 1-7 may be called an 'informal Principia system,' since all the propositions, both 'formal' and 'informal,' in Section A of the Principia are deducible from these seven postulates."

But a glance at Postulates 1-7 shows that the postulates are all satisfied when the class $K$ is empty. Hence, the postulates cannot yield the proposition $Q$ above. Hence, Huntington's "informal Principia system," as given by Postulates 1-7, is inadequate for the classic logic of classes and for the classic logic of propositions. $\dagger$

$\dagger$ The inadequacy in question concerns only the "informal Principia system" given by Postulates 1-7. A two-element Boolean algebra would be a logic of propositions and would, in the sense of Huntington's definition, also be an "informal Principia system," though the postulates for such an algebra would not be equivalent to Postulates 1-7.

Huntington says (p. 302): 'Hence, an 'informal Principia system,' as above defined, contains only two 'non-equivalent' elements." In view of the fact that $K$ may be empty, only two must be taken to mean at most two. 
6. Remark on "Informal" Systems. There are two apparently insurmountable difficulties that must be overcome before one can obtain a logical system which would represent the combined "formal" and "informal" Section A, and which would yield the classic logic of propositions. One difficulty is that the combined "formal" and "informal" Section A must not contain existence propositions. For, the Principia (p. xiv) says: "General propositions of the form ' $(p) \cdot f p$ ' do occur in Section A, but ' $(\exists p) \cdot f p$ ' does not occur." This "informal" statement does not permit in Section A such propositions of the logic of propositions as proposition $Q$ above $(\$ 4) . \dagger$

The second difficulty is that the combined "formal" and "informal" Section A contains inconsistencies. For instance, the Princıpia (p. 93) says: "If $p$ is any proposition, the proposition 'not- $p$,' or ' $p$ is false,' will be represented by ' $\sim p$ '." The Principia here identifies " $\sim p$," which is an "elementary" proposition, by $* 1.71$, with " $\vdash \cdot \sim p$," which is a non-"elementary" proposition. Or, to put the matter a little differently, the Principia here identifies " $p$ " with " $\vdash \cdot p$," and thus violates the distinction between " $p$ " and " $\vdash \cdot p$ " which it carefully made earlier (p. 92). $\ddagger$

There are minor difficulties connected with the finding of a logical system equivalent to the combined "formal" and "informal" Section A. For instance, precisely what are all the "informal" statements in Section A? May (or must) "informal" statements outside Section A be included in the proposed system, provided such outside statements have a bearing on Section A? If so, precisely what are these statements?

The University of California

$\dagger$ In my Paper 1 (loc. cit.), I transcribed the Principia proposition *1.1 in the form 1.1, which is an existence proposition. In this transcription, however, I was concerned only with the "formal" Section A. I saw an existence notion implied in *1.1, and I disregarded Principia, p. xiv.

$\ddagger$ Huntington reconciles " $a \supset b$ " the element with " $a \supset b$ " the relation, "which are not in precise agreement," by the following definitions.

Definition. $a \supset b$ means $a^{\prime}+b$;

Definition. $(a$ implies $b)$ means $\left(a^{\prime}+b\right.$ is in $\left.T\right)$.

It seems to me that these definitions merely recognize the disagreement. The definitions merely say that " $a \supset b$ " is an element, namely $a^{\prime}+b$, and that " $a$ implies $b$ " is a relation, namely " $\vdash \cdot a^{\prime}+b . "$ 